\title{
A Reciprocal $g$-Derivatives of 2-nd Type and its Properties
}

\author{
M. M. Pahirya \\ Mukachevo state university, Mukachevo, Ukraine \\ pahirya@gmail.com
}

Keywords: Approximation of functions of complex variables, quasi-reciprocal functional continued fractions, reciprocal $g$-derivatives of 2-nd type, functional formula of Thiele type.

Abstract. A new type of functional reciprocal derivatives called reciprocal $g$-derivatives of $2-$ nd type are introduced in the consideration. Analogue of Thiele formula for quasi- reciprocal functional continued fractions has been proposed.

\section{Introduction}

It is well known that the Thiele formula is an analogue of Taylor formula in the theory of continued fractions [1]. Reciprocal derivatives are used in the Thiele formula. Reciprocal derivatives of 2-nd type are introduced in [2]. Reciprocal $g$-derivatives have been studied in [3].

Reciprocal $g$-derivatives of 2-nd type have been introduced and some of its properties have been established in this paper. Analogue of Thiele type formula for quasi-reciprocal functional continued fraction has been obtained.

\section{Quasi-reciprocal functional interpolation Thiele-like continued fraction}

Let basic-function $g(z)$ is one-sheeted function on $\mathcal{Z}$, function $f(z)$ is defined on the compact $\mathcal{Z} \subset \mathbb{C}$ and is determined in the points of set

$$
\mathbf{Z}=\left\{z_{i}: z_{i} \in \mathcal{Z}, z_{i} \neq z_{j}, i, j=0,1, \ldots, n\right\}, \quad w_{i}=f\left(z_{i}\right), \quad i=0,1, \ldots, n .
$$

We define the elements of sequences $\left\{v_{k}(g ; z)\right\}$ and $\left\{V_{k}(g ; z)\right\}$ as follows

$$
\begin{gathered}
f(z)=\frac{1}{v_{0}(g ; z)}, v_{k}(g ; z)=v_{k}\left(g ; z_{k}\right)+\frac{g(z)-g\left(z_{k}\right)}{v_{k+1}(g ; z)}, k=0,1, \ldots, n, \\
V_{0}(g ; z)=v_{0}(g ; z), \quad V_{k}(g ; z)=V_{k-1}\left(g ; v_{k}(g ; z)\right), \quad k=1,2, \ldots, n .
\end{gathered}
$$

We have

$$
f(z)=\frac{1}{V_{n}(g ; z)}=\left(v_{0}\left(g ; z_{0}\right)+\frac{g(z)-g\left(z_{0}\right)}{v_{1}\left(g ; z_{1}\right)}+\cdots+\frac{g(z)-g\left(z_{n-1}\right)}{v_{n}\left(g ; z_{n}\right)}+\frac{g(z)-g\left(z_{n}\right)}{v_{n+1}(g ; z)}\right)^{-1} .
$$

We denote $d_{k}^{(g)}=v_{k}\left(g ; z_{k}\right), k=0,1, \ldots, n$, cast out $\left(g(z)-g\left(z_{n}\right)\right) / v_{n+1}(g ; z)$ and then we have functional continued fraction of the form

$$
D_{n}^{(t)}(g ; z)=\left(d_{0}^{(g)}+\frac{g(z)-g\left(z_{0}\right)}{d_{1}^{(g)}}+\frac{g(z)-g\left(z_{1}\right)}{d_{2}^{(g)}}+\cdots+\frac{g(z)-g\left(z_{n-1}\right)}{d_{n}^{(g)}}\right)^{-1} .
$$

We used forward or backward recurrence algorithm [4] and we assigne continued fraction (3) ration of two generalized polynomials of $g(z)$

$$
D_{n}^{(t)}(g ; z)=\frac{P_{n}^{(t)}(g ; z)}{Q_{n}^{(t)}(g ; z)}=\left(d_{0}^{(g)}+\frac{g(z)-g\left(z_{0}\right)}{d_{1}^{(g)}}+\cdots+\frac{g(z)-g\left(z_{n-1}\right)}{d_{n}^{(g)}}\right)^{-1} .
$$


Definition 1. If in the points of set (1) interpolation conditions $w_{i}=D_{n}^{(t)}\left(g ; z_{i}\right), i=0,1, \ldots, n$, are valid then continued fraction (4) is named quasi-reciprocal functional of Thiele-type continued fraction (T-QFICF).

Theorem 2 [3]. Coefficients of T-QFICF (4) are determined by the values of function $f(z)$ in points from $\mathbf{Z}$ with the help of recurrence relation in the form of finite continued fraction

$$
d_{k}^{(g)}=\frac{g\left(z_{k}\right)-g\left(z_{k-1}\right)}{-d_{k-1}^{(g)}}+\cdots+\frac{g\left(z_{k}\right)-g\left(z_{1}\right)}{-d_{1}^{(g)}}+\frac{g\left(z_{k}\right)-g\left(z_{0}\right)}{1 / w_{k}-d_{0}^{(g)}}, d_{0}^{(g)}=\frac{1}{w_{0}}, \quad k=1,2, \ldots, n .
$$

It is easy to prove the following statement.

Theorem 3. Canonical numerate $P_{n}^{(t)}(g ; z)$ and canonical denominate $Q_{n}^{(t)}(g ; z)$ of T-QFICF (4) are generalized polynomials of $g(z)$, degree of generalized polynomial satisfies the inequalities

$$
\operatorname{deg} P_{n}^{(t)}(g ; z) \leq[n / 2], \quad \operatorname{deg} Q_{n}^{(t)}(g ; z) \leq[(n+1) / 2]
$$

\section{Reciprocal divided $g$-difference of 2 -nd type}

From formula (2) follows

$$
v_{0}(g ; z)=\frac{1}{f(z)}, \quad v_{k+1}(g ; z)=\frac{g(z)-g\left(z_{k}\right)}{v_{k}(g ; z)-v_{k}\left(g ; z_{k}\right)}, \quad k=0,1, \ldots
$$

Let's introduce into consideration reciprocal divided $g$-difference of 2 -nd type of $k$-th order with the help of following relation

$$
\begin{aligned}
\Phi_{k}^{(2)}\left[g ; z_{0}, \ldots, z_{k} ; f\right] & =\frac{g\left(z_{k}\right)-g\left(z_{k-1}\right)}{\Phi_{k-1}^{(2)}\left[g ; z_{0}, \ldots, z_{k-2}, z_{k} ; f\right]-\Phi_{k-1}^{(2)}\left[g ; z_{0}, \ldots, z_{k-1} ; f\right]}, \quad k=1,2, \ldots, \\
\Phi_{0}^{(2)}[g ; z ; f] & =1 / f(z) .
\end{aligned}
$$

Then $d_{k}^{(g)}=v_{k}\left(g ; z_{k}\right)=\Phi_{k}^{(2)}\left[g ; z_{0}, z_{1}, \ldots, z_{k} ; f\right]$.

The reciprocal divided $g$-difference of 2 -nd type of $k$-th order is determined by the interpolation notes $z_{0}, z_{1}, \ldots, z_{k}$ and values of the function $f(z)$ at these nodes on the one hand and it is a symmetric function of only the last two of its arguments $z_{k-1}$ and $z_{k}$ on the other hand.

Now, we form the reciprocal $g$-differences of 2 -nd type of $k$-th order using the following relation

$$
\begin{gathered}
\varrho_{0}^{(2)}=\varrho_{0}^{(2)}\left[g ; z_{0} ; f\right]=\Phi_{0}^{(2)}\left[g ; z_{0} ; f\right]=\frac{1}{f\left(z_{0}\right)}, \\
\varrho_{1}^{(2)}=\varrho_{0}^{(2)}\left[g ; z_{0}, z_{1} ; f\right]=\Phi_{1}^{(2)}\left[g ; z_{0}, z_{1} ; f\right]=\frac{g\left(z_{1}\right)-g\left(z_{0}\right)}{\varrho_{0}^{(2)}\left[g ; z_{1} ; f\right]-\varrho_{0}^{(2)}\left[g ; z_{0} ; f\right]}, \\
\varrho_{k}^{(2)}=\varrho_{k}^{(2)}\left[g ; z_{0}, z_{1}, \ldots, z_{k} ; f\right]=\sum_{i=0}^{[k / 2]} \Phi_{k-2 i}^{(2)}\left[g ; z_{0}, z_{1}, \ldots, z_{k-2 i} ; f\right], \quad k=2,3, \ldots, n .
\end{gathered}
$$

From the formulas (5)-(7) it directly follows that reciprocal $g$-differences of 2 -nd type satisfy the recurrence relation

$$
\begin{aligned}
& \varrho_{k}^{(2)}\left[g ; z_{0}, \ldots, z_{k} ; f\right]=\varrho_{k-2}^{(2)}\left[g ; z_{0}, \ldots, z_{k-2} ; f\right]+ \\
& +\frac{g\left(z_{k}\right)-g\left(z_{k-1}\right)}{\varrho_{k-1}^{(2)}\left[g ; z_{0}, \ldots, z_{k-2}, z_{k} ; f\right]-\varrho_{k-1}^{(2)}\left[g ; z_{0}, \ldots, z_{k-1} ; f\right]}, k=2,3, \ldots, \\
& \varrho_{0}^{(2)}\left[g ; z_{0} ; f\right]=\frac{1}{f\left(z_{0}\right)}, \quad \varrho_{1}^{(2)}\left[g ; z_{0}, z_{1} ; f\right]=\frac{g\left(z_{1}\right)-g\left(z_{0}\right)}{\varrho_{0}^{(2)}\left[g ; z_{1} ; f\right]-\varrho_{0}^{(2)}\left[g ; z_{0} ; f\right]} .
\end{aligned}
$$


The coefficients of T-QFICF (4) $d_{k}^{(g)}$, when $k=0,1, \ldots, n$, are determined through reciprocal $g$-differences of 2 -nd type in the following way

$$
\begin{aligned}
& d_{0}^{(g)}=\varrho_{0}^{(2)}=\varrho_{0}^{(2)}\left[g ; z_{0} ; f\right], \quad d_{1}^{(g)}=\varrho_{1}^{(2)}=\varrho_{1}^{(2)}\left[g ; z_{0}, z_{1} ; f\right], \\
& d_{k}^{(g)}=\varrho_{k}^{(2)}-\varrho_{k-2}^{(2)}=\varrho_{k}^{(2)}\left[g ; z_{0}, \ldots, z_{k} ; f\right]-\varrho_{k-2}^{(2)}\left[g ; z_{0}, \ldots, z_{k-2} ; f\right], \quad k=2,3, \ldots, n .
\end{aligned}
$$

In this case, the T-QFICF (4) can be rewritten as

$$
D_{n}^{(t)}(g ; z)=\left(\varrho_{0}^{(2)}+\frac{g(z)-g\left(z_{0}\right)}{\varrho_{1}^{(2)}}+\frac{g(z)-g\left(z_{1}\right)}{\varrho_{2}^{(2)}-\varrho_{0}^{(2)}}+\cdots+\frac{g(z)-g\left(z_{n-1}\right)}{\varrho_{n}^{(2)}-\varrho_{n-2}^{(2)}}\right)^{-1} .
$$

In [3] it has been proven that

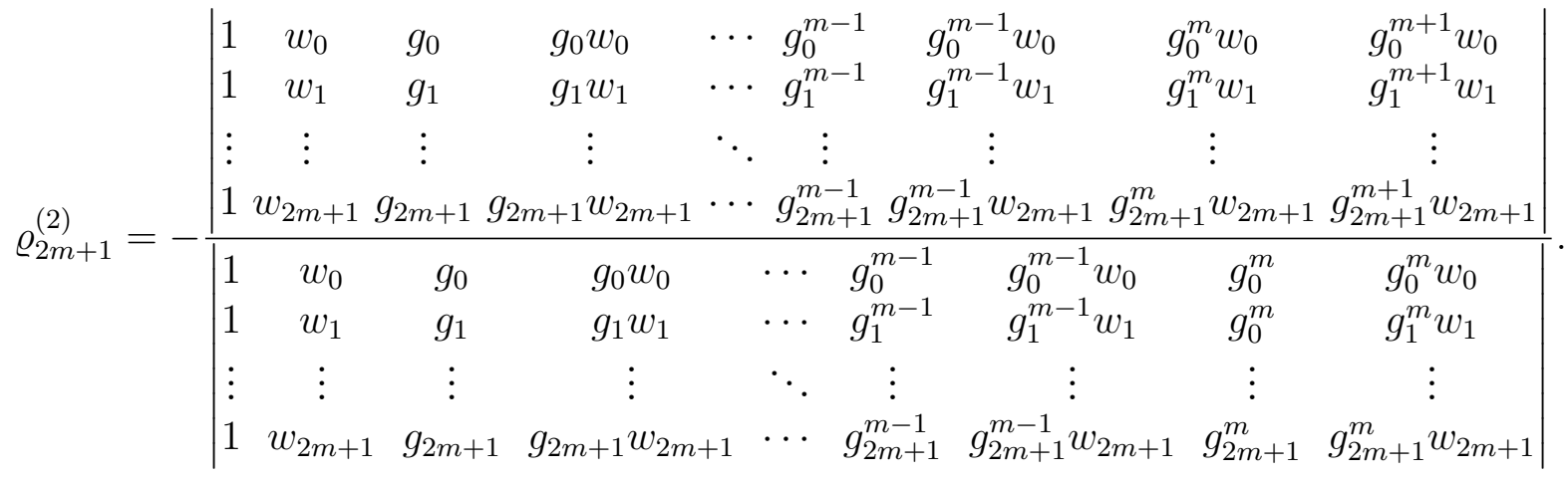

$$
\begin{aligned}
& \varrho_{2 m}^{(2)}=\frac{\left|\begin{array}{cccccccc}
1 & w_{0} & g_{0} & g_{0} w_{0} & \cdots & g_{0}^{m-1} & g_{0}^{m-1} w_{0} & g_{0}^{m} \\
1 & w_{1} & g_{1} & g_{1} w_{1} & \cdots & g_{1}^{m-1} & g_{1}^{m-1} w_{1} & g_{1}^{m} \\
\vdots & \vdots & \vdots & \vdots & \ddots & \vdots & \vdots & \vdots \\
1 & w_{2 m} & g_{2 m} & g_{2 m} w_{2 m} & \cdots & g_{2 m}^{m-1} & g_{2 m}^{m-1} w_{2 m} & g_{2 m}^{m}
\end{array}\right|}{\left|\begin{array}{cccccccc}
1 & w_{0} & g_{0} & g_{0} w_{0} & \cdots & g_{0}^{m-1} & g_{0}^{m-1} w_{0} & g_{0}^{m} w_{0} \\
1 & w_{1} & g_{1} & g_{1} w_{1} & \cdots & g_{1}^{m-1} & g_{1}^{m-1} w_{1} & g_{1}^{m} w_{1} \\
\vdots & \vdots & \vdots & \vdots & \ddots & \vdots & \vdots & \vdots \\
1 & w_{2 m} & g_{2 m} & g_{2 m} w_{2 m} & \cdots & g_{2 m}^{m-1} & g_{2 m}^{m-1} w_{2 m} & g_{2 m}^{m} w_{2 m}
\end{array}\right|} \quad m=1,2, \ldots,
\end{aligned}
$$

where $w_{i}=f\left(z_{i}\right), g_{i}=g\left(z_{i}\right), i=0,1, \ldots, n$.

From (11)-(12) it follows that reciprocal $g$-differences of 2-nd type are symmetric function of all its arguments.

\section{Reciprocal $g$-derivatives of 2-nd type}

In constructing the T-QFICF (4) it was assumed that all interpolation nodes $z_{i}, i=0, \ldots, n$, are different. We now consider the limiting case when all the notes or some of them tend to same value $z \in \mathcal{Z}$.

Definition 4. If there is limit, finite or infinite value, reciprocal $g$-difference of 2-nd type of $k$-th order (7), when interpolation nodes $z_{0}, z_{1}, \ldots, z_{k} \in \mathbf{Z}$ tend to some $z \in \mathcal{Z}$, then the limiting value is called reciprocal $g$-derivative of 2 -nd type of $k$-th order. The reciprocal $g$-derivative of 2 -nd type of $k$-th order at the point $z \in \mathcal{Z}$ is denoted as ${ }^{[k]} f_{g}(z)$.

We have from the definition that

$$
{ }^{[k]} f_{g}(z)=\varrho_{k}^{(2)}[g ; \underbrace{z, \ldots, z}_{k+1} ; f]=\lim _{z_{0}, z_{1}, \ldots, z_{k} \rightarrow z} \varrho_{k}^{(2)}\left[g ; z_{0}, z_{1}, \ldots, z_{k} ; f\right] .
$$


From (5), (6) and (13) follows

$$
\begin{aligned}
& { }^{[1]} f_{g}(z)=\varrho_{1}^{(2)}[g ; z, z ; f]=\lim _{z_{0}, z_{1} \rightarrow z} \frac{\left|\begin{array}{ll}
f\left(z_{0}\right) & g\left(z_{0}\right) f\left(z_{0}\right) \\
f\left(z_{1}\right) & g\left(z_{1}\right) f\left(z_{1}\right)
\end{array}\right|}{-\left|\begin{array}{cc}
1 & f\left(z_{0}\right) \\
1 & f\left(z_{1}\right)
\end{array}\right|}=\lim _{\Delta z \rightarrow 0} \frac{\left|\begin{array}{cc}
f(z) & g(z) f(z) \\
f(z+\Delta z) & g(z+\Delta z) f(z+\Delta z)
\end{array}\right|}{-\left|\begin{array}{cc}
1 & f(z) \\
1 & f(z+\Delta z)
\end{array}\right|}= \\
& =\lim _{\Delta z \rightarrow 0} \frac{\left|\begin{array}{cc}
f(z) & g(z) f(z) \\
\frac{f(z+\Delta z)-f(z)}{\Delta z} & \frac{g(z+\Delta z) f(z+\Delta z)-g(z) f(z)}{\Delta z}
\end{array}\right|}{-\left|\begin{array}{cc}
1 & f(z) \\
0 & \frac{f(z+\Delta z)-f(z)}{\Delta z}
\end{array}\right|}=-\frac{\left|\begin{array}{cc}
f(z) & g(z) f(z) \\
f^{\prime}(z) & (g(z) f(z))^{\prime}
\end{array}\right|}{\left|\begin{array}{ll}
1 & f(z) \\
0 & f^{\prime}(z)
\end{array}\right|}=-\frac{f^{2}(z) g^{\prime}(z)}{f^{\prime}(z)} .
\end{aligned}
$$

Similarly, we get from the formula (12) and (13) when $m=1$ that

$$
\begin{aligned}
& { }^{[2]} f_{g}(z)=\varrho_{2}^{(2)}[g ; z, z, z ; f]=\lim _{z_{0}, z_{1}, z_{2} \rightarrow z} \frac{\left|\begin{array}{ccc}
1 & f\left(z_{0}\right) & g\left(z_{0}\right) \\
1 & f\left(z_{1}\right) & g\left(z_{1}\right) \\
1 & f\left(z_{2}\right) & g\left(z_{2}\right)
\end{array}\right|}{\left|\begin{array}{ccc}
1 & f\left(z_{0}\right) & g\left(z_{0}\right) f\left(z_{0}\right) \\
1 & f\left(z_{1}\right) & g\left(z_{1}\right) f\left(z_{1}\right) \\
1 & f\left(z_{2}\right) & g\left(z_{2}\right) f\left(z_{2}\right)
\end{array}\right|}=
\end{aligned}
$$

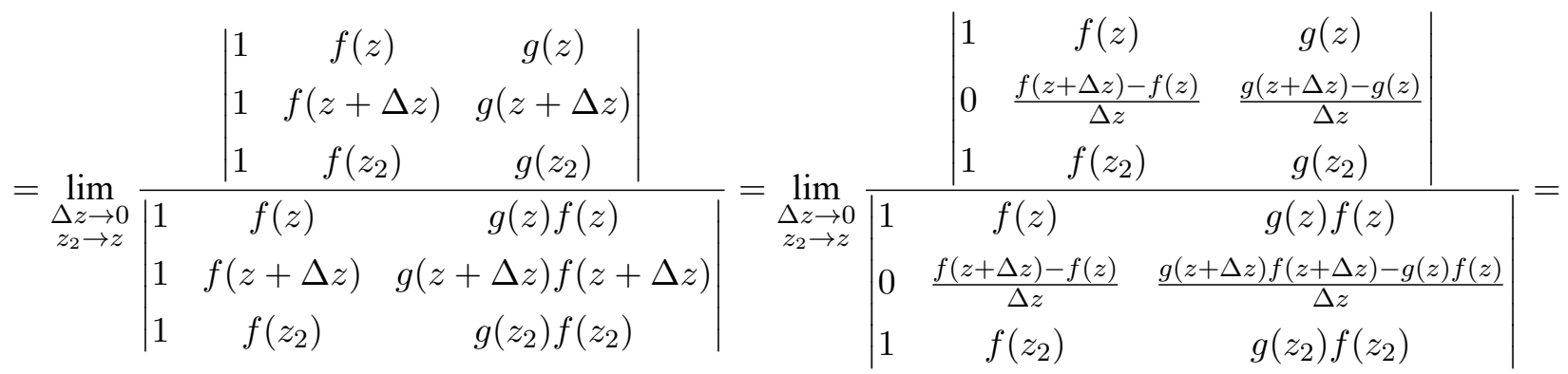

$$
\begin{aligned}
& =\lim _{\Delta z \rightarrow 0} \frac{\left|\begin{array}{ccc}
1 & f(z) & g(z) \\
0 & f^{\prime}(z) & g^{\prime}(z) \\
1 & f(z+\Delta z) & g(z+\Delta z)
\end{array}\right|}{\left|\begin{array}{ccc}
1 & f(z) & g(z) f(z) \\
0 & f^{\prime}(z) & (g(z) f(z))^{\prime} \\
1 & f(z+\Delta z) & g(z+\Delta z) f(z+\Delta z)
\end{array}\right|}=
\end{aligned}
$$

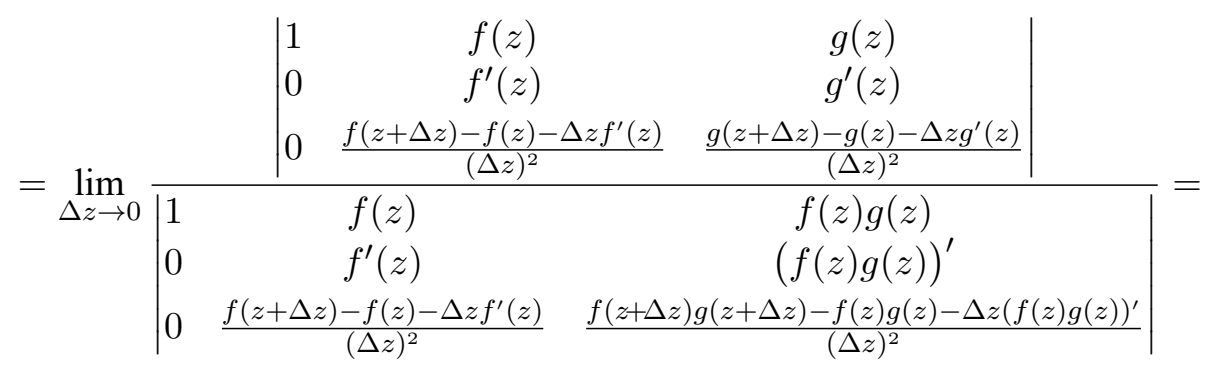

$$
\begin{aligned}
& =\frac{\left|\begin{array}{ll}
f^{\prime}(z) & g^{\prime}(z) \\
\frac{f^{\prime \prime}(z)}{2 !} & \frac{g^{\prime \prime}(z)}{2 !}
\end{array}\right|}{\left|\begin{array}{ll}
f^{\prime}(z) & (f(z) g(z))^{\prime} \\
\frac{f^{\prime \prime}(z)}{2 !} & \frac{(f(z) g(z))^{\prime \prime}}{2 !}
\end{array}\right|}=\frac{\left|\begin{array}{ll}
f^{\prime}(z) & g^{\prime}(z) \\
f^{\prime \prime}(z) & g^{\prime \prime}(z)
\end{array}\right|}{\left|\begin{array}{ll}
f^{\prime}(z) & (f(z) g(z))^{\prime} \\
f^{\prime \prime}(z) & (f(z) g(z))^{\prime \prime}
\end{array}\right|} .
\end{aligned}
$$


We obtain a formula for reciprocal $g$-derivative of 2-nd type of $k$ order in general. We consider two cases: a) $k=2 m$; b) $k=2 m+1$.

a) Let $k=2 m$. According to the formulas (12) and (13) we have

$$
\begin{aligned}
& { }^{[2 m]} f_{g}(z)=\varrho_{2 m}^{(2)}[g ; \underbrace{z, z, \cdots, z}_{2 m+1} ; f]=\lim _{z_{0}, \cdots, z_{2 m} \rightarrow z} \varrho_{2 m}\left[g ; z_{0}, z_{1}, \cdots, z_{2 m} ; f\right]=
\end{aligned}
$$

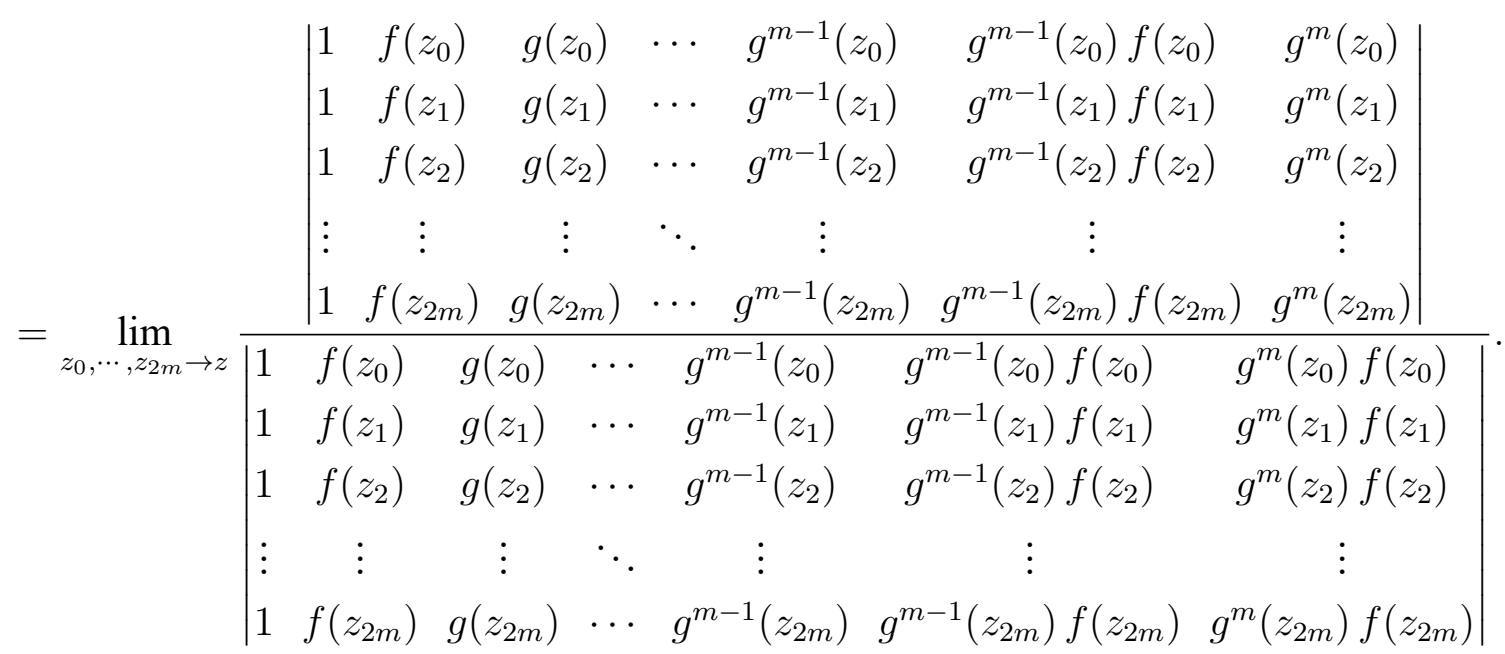

We will gradually pass to the limit in the determinants of the numerator and the denominator. In the first step, we substitute $z$ instead of $z_{0}$ and substitute $(z+\Delta z)$ instead of $z_{1}$, we subtract 1-st row from 2-th row of the determinants, we divide 2-th row by $\Delta z$ and we pass to the limit as $\Delta z \rightarrow 0$. In the second step, we substitute $z+\Delta z$ instead of $z_{2}$, we subtract 1-st row and 2-th row multiplied by $\Delta z$ from 3-th row, we divide 3 -th row by $(\Delta z)^{2}$ and we pass to the limits as $\Delta z \rightarrow 0$. And so on. In the $i$-th step, $i=1,2, \ldots, 2 m$, we substitute $z+\Delta z$ instead of $z_{i}$, we subtract 1-st row, 2-th row multiplied by $\Delta z, 3-$ th row multiplied by $(\Delta z)^{2}$ and so on, $i$-th row multiplied by $(\Delta z)^{i-1}$ from $(i+1)$-th row, we divide obtained $(i+1)$-th row by $(\Delta z)^{i}$ and we pass to limit as $\Delta z \rightarrow 0$. After $(2 m)$ such steps we finally get

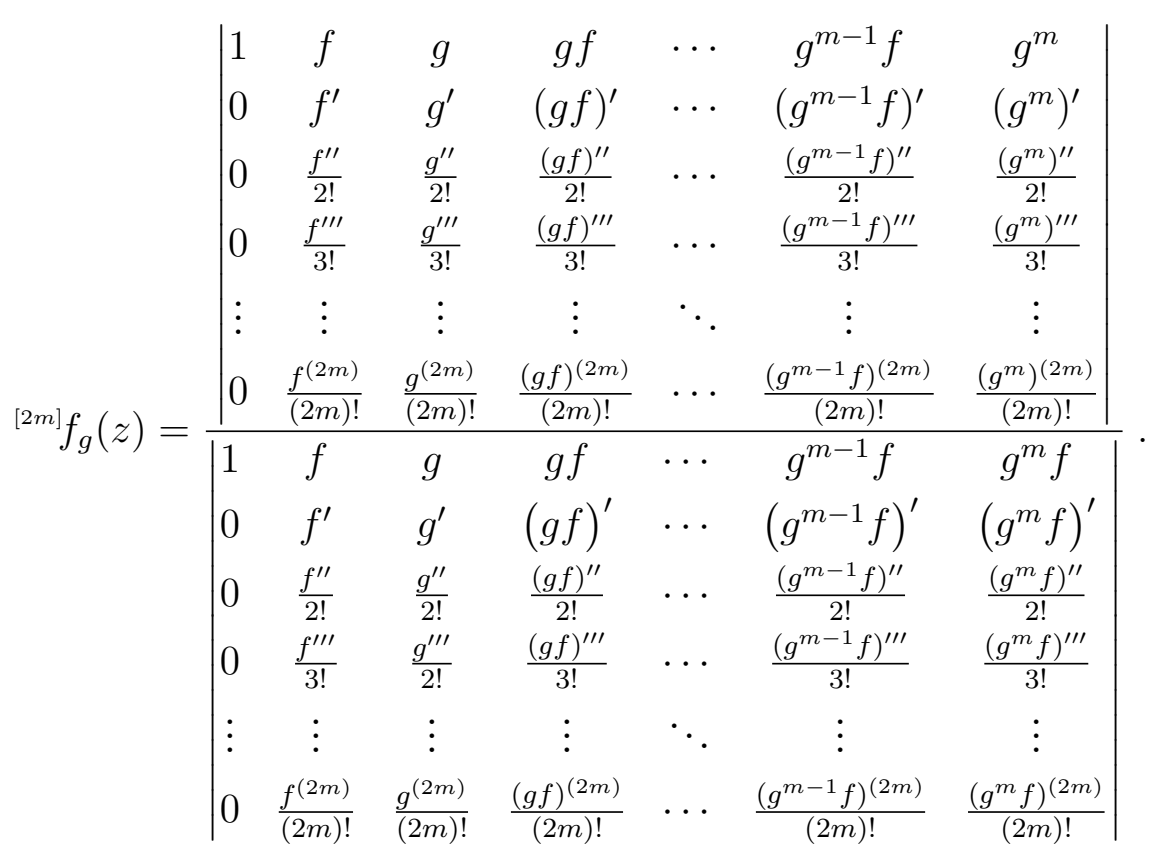


After the obvious simplifications we have

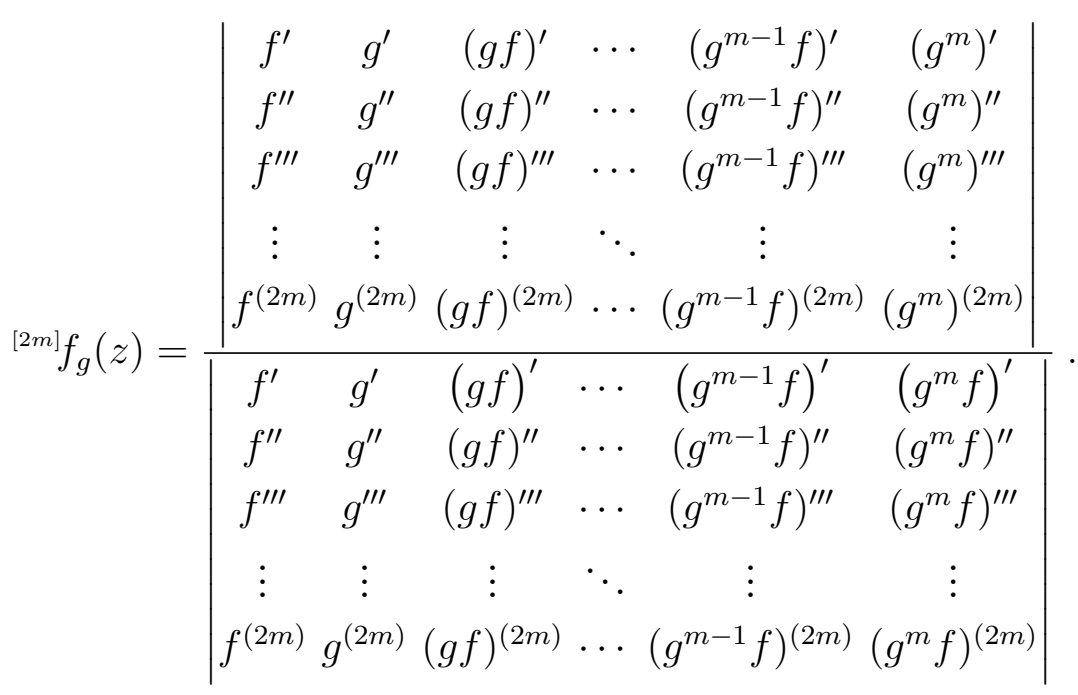

b) In the case where $k=2 m+1$ we similarly have that

$$
\begin{aligned}
& { }^{[2 m+1]} f_{g}(z)=\varrho_{2 m+1}^{(2)}[g ; \underbrace{z, z, \cdots, z}_{2 m+2} ; f]=\lim _{z_{0}, \cdots, z_{2 m+1} \rightarrow z} \varrho_{2 m+1}\left[g ; z_{0}, z_{1}, \cdots, z_{2 m+1} ; f\right]=
\end{aligned}
$$

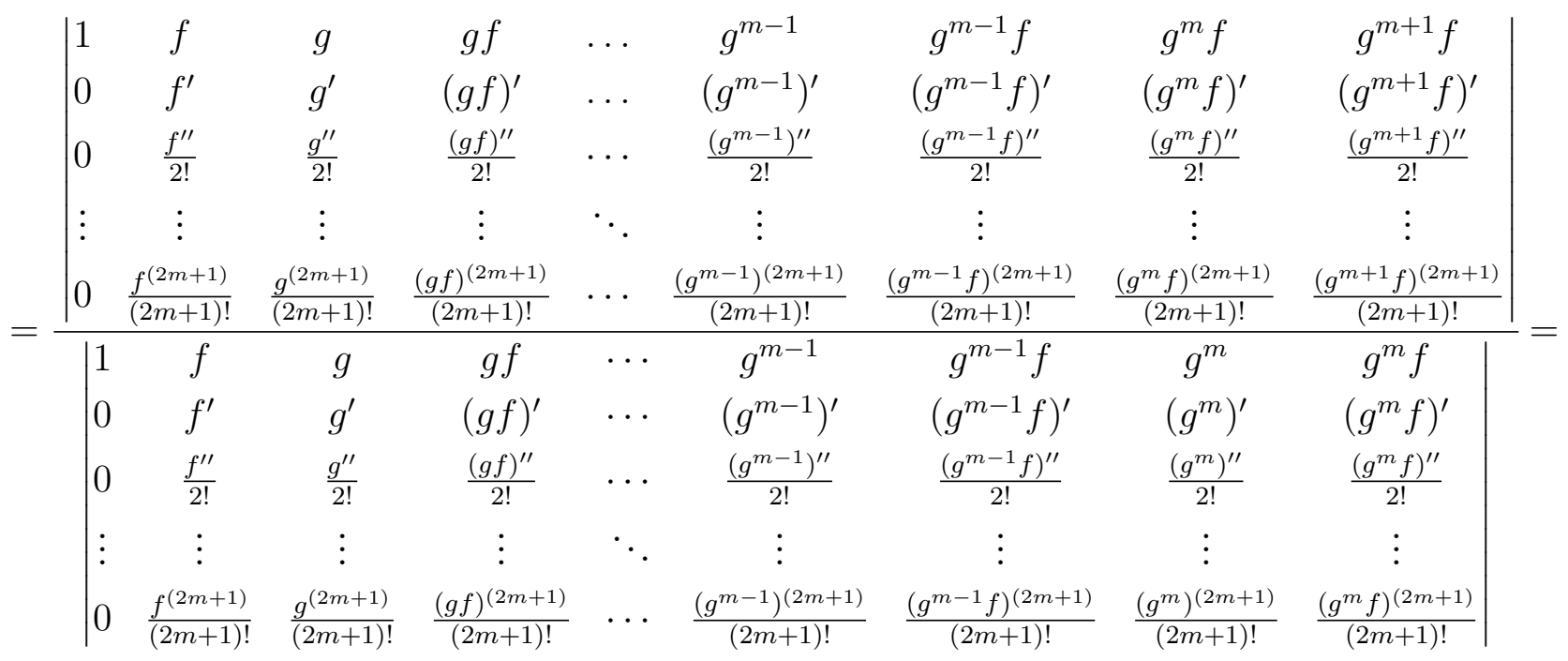

$$
\begin{aligned}
& \begin{array}{|cccccccc}
f & g & g f & \ldots & g^{m-1} & g^{m-1} f & g^{m} f & g^{m+1} f \\
f^{\prime} & g^{\prime} & (g f)^{\prime} & \ldots & \left(g^{m-1}\right)^{\prime} & \left(g^{m-1} f\right)^{\prime} & \left(g^{m} f\right)^{\prime} & \left(g^{m+1} f\right)^{\prime} \\
f^{\prime \prime} & g^{\prime \prime} & (g f)^{\prime \prime} & \ldots & \left(g^{m-1}\right)^{\prime \prime} & \left(g^{m-1} f\right)^{\prime \prime} & \left(g^{m} f\right)^{\prime \prime} & \left(g^{m+1} f\right)^{\prime \prime}
\end{array}
\end{aligned}
$$

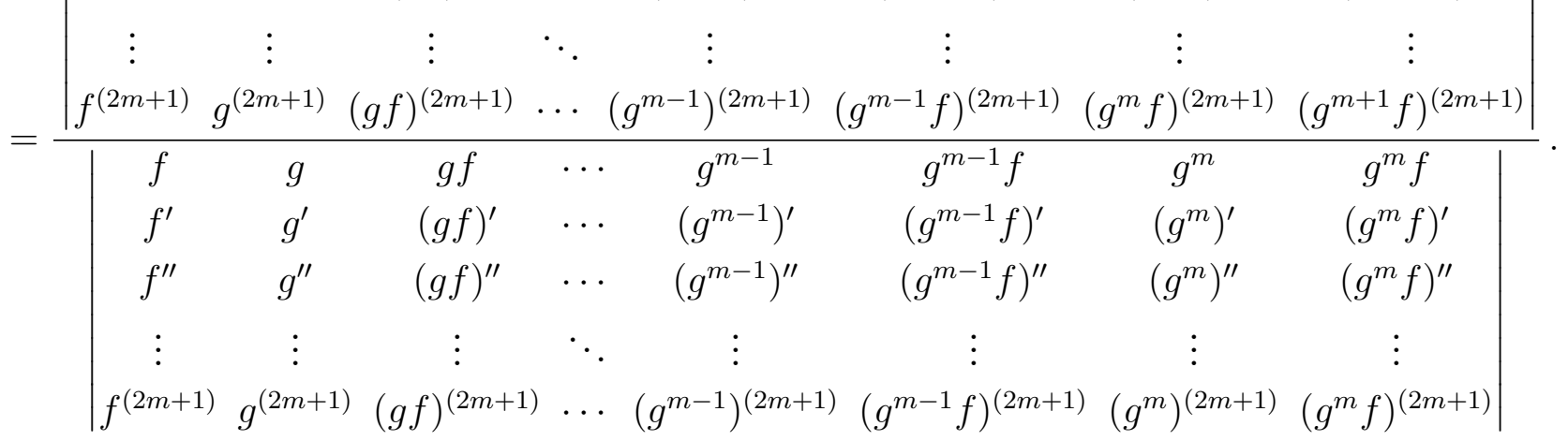

Arguments of functions $f(z), g(z)$ and their derivatives are missed in all formulas.

Thus the following statement has been proved. 
Theorem A Iffor some value $m$ determinants

$$
\begin{gathered}
F_{m}^{(1)}(z)=\left|\begin{array}{cccccc}
f^{\prime} & g^{\prime} & (g f)^{\prime} & \cdots & \left(g^{m-1} f\right)^{\prime} & \left(g^{m}\right)^{\prime} \\
f^{\prime \prime} & g^{\prime \prime} & (g f)^{\prime \prime} & \cdots & \left(g^{m-1} f\right)^{\prime \prime} & \left(g^{m}\right)^{\prime \prime} \\
\vdots & \vdots & \vdots & \ddots & \vdots & \vdots \\
f^{(2 m)} & g^{(2 m)} & (g f)^{(2 m)} & \cdots & \left(g^{m-1} f\right)^{(2 m)} & \left(g^{m}\right)^{(2 m)}
\end{array}\right|, \\
F_{m}^{(2)}(z)=\left|\begin{array}{cccccc}
f^{\prime} & g^{\prime} & (g f)^{\prime} & \cdots & \left(g^{m-1} f\right)^{\prime} & \left(g^{m} f\right)^{\prime} \\
f^{\prime \prime} & g^{\prime \prime} & (g f)^{\prime \prime} & \cdots & \left(g^{m-1} f\right)^{\prime \prime} & \left(g^{m} f\right)^{\prime \prime} \\
\vdots & \vdots & \vdots & \ddots & \vdots & \vdots \\
f^{(2 m)} & g^{(2 m)} & (g f)^{(2 m)} & \cdots & \left(g^{m-1} f\right)^{(2 m)} & \left(g^{m} f\right)^{(2 m)}
\end{array}\right|
\end{gathered}
$$

different from zero at some point $z \in \mathcal{Z}$ then at this point function $f(z)$ has reciprocal $g$-derivative of 2-nd type of $(2 m)$-th order and

$$
{ }^{[2]} f_{g}(z)=\frac{\left|\begin{array}{ll}
f^{\prime} & g^{\prime} \\
f^{\prime \prime} & g^{\prime \prime}
\end{array}\right|}{\left|\begin{array}{ll}
f^{\prime} & (f g)^{\prime} \\
f^{\prime \prime} & (f g)^{\prime \prime}
\end{array}\right|}, \quad{ }^{[2 m]} f_{g}(z)=\frac{F_{m}^{(1)}(z)}{F_{m}^{(2)}(z)}, \quad m=2,3, \ldots
$$

(B) If for some value $m$ determinants

$$
\begin{gathered}
F_{m}^{(3)}(z)=\left|\begin{array}{cccccccc}
f & g & g f & \ldots & g^{m-1} & g^{m-1} f & g^{m} f & g^{m+1} f \\
f^{\prime} & g^{\prime} & (g f)^{\prime} & \ldots & \left(g^{m-1}\right)^{\prime} & \left(g^{m-1} f\right)^{\prime} & \left(g^{m} f\right)^{\prime} & \left(g^{m+1} f\right)^{\prime} \\
f^{\prime \prime} & g^{\prime \prime} & (g f)^{\prime \prime} & \cdots & \left(g^{m-1}\right)^{\prime \prime} & \left(g^{m-1} f\right)^{\prime \prime} & \left(g^{m} f\right)^{\prime \prime} & \left(g^{m+1} f\right)^{\prime \prime} \\
\vdots & \vdots & \vdots & \ddots & \vdots & \vdots & \vdots & \vdots \\
f^{(k)} & g^{(k)} & (g f)^{(k)} & \ldots & \left(g^{m-1}\right)^{(k)} & \left(g^{m-1} f\right)^{(k)} & \left(g^{m} f\right)^{(k)} & \left(g^{m+1} f\right)^{(k)}
\end{array}\right|, \\
F_{m}^{(4)}(z)=\left|\begin{array}{cccccccc}
f & g & g f & \ldots & g^{m-1} & g^{m-1} f & g^{m} & g^{m} f \\
f^{\prime} & g^{\prime} & (g f)^{\prime} & \ldots & \left(g^{m-1}\right)^{\prime} & \left(g^{m-1} f\right)^{\prime} & \left(g^{m}\right)^{\prime} & \left(g^{m} f\right)^{\prime} \\
f^{\prime \prime} & g^{\prime \prime} & (g f)^{\prime \prime} & \ldots & \left(g^{m-1}\right)^{\prime \prime} & \left(g^{m-1} f\right)^{\prime \prime} & \left(g^{m}\right)^{\prime \prime} & \left(g^{m} f\right)^{\prime \prime} \\
\vdots & \vdots & \vdots & \ddots & \vdots & \vdots & \vdots & \vdots \\
f^{(k)} & g^{(k)} & (g f)^{(k)} & \ldots & \left(g^{m-1}\right)^{(k)} & \left(g^{m-1} f\right)^{(k)} & \left(g^{m}\right)^{(k)} & \left(g^{m} f\right)^{(k)}
\end{array}\right|, \quad k=2 m+1,
\end{gathered}
$$

different from zero at some point $z \in \mathcal{Z}$ then at this point function $f(z)$ has reciprocal $g$-derivative of 2-nd type of $(2 m+1)$-th order and

$$
{ }^{[1]} f_{g}(z)=-\frac{f^{2}(z) g^{\prime}(z)}{f^{\prime}(z)}, \quad{ }^{[2 m+1]} f_{g}(z)=\frac{F_{m}^{(3)}(z)}{F_{m}^{(4)}(z)} \quad m=1,2, \ldots
$$

Remark. If $g(z)=z$ then formulas (15) and (16) after obvious simplifications will coincide with the formulas obtained in [2].

Similarly, as was done in the book [3], from (8) we get recurrence formula for determining reciprocal $g$-derivatives of 2-nd type 


$$
\begin{aligned}
& { }^{[k]} f_{g}(z)=\frac{k g^{\prime}(z)}{\left({ }^{[k-1]} f_{g}(z)\right)^{\prime}}+{ }^{[k-2]} f_{g}(z), \quad k=2,3, \ldots, \\
& { }^{[0]} f_{g}(z)=\frac{1}{f(z)}, \quad{ }^{[1]} f_{g}(z)=\frac{-f^{2}(z) g^{\prime}(z)}{f^{\prime}(z)},
\end{aligned}
$$

From formulas (9), (10) and (13) follows that in neighborhood of point $z=z_{*}$ has place a functional formula of Thiele type

$$
\begin{gathered}
f(z)=\left(\frac{1}{f\left(z_{*}\right)}+\frac{g(z)-g\left(z_{*}\right)}{{ }^{[1]} f_{g}\left(z_{*}\right)}+\frac{g(z)-g\left(z_{*}\right)}{2 g^{\prime}(z) /\left({ }^{[1]} f_{g}\left(z_{*}\right)\right)^{\prime}}+\frac{g(z)-g\left(z_{*}\right)}{3 g^{\prime}(z) /\left({ }^{[2]} f_{g}\left(z_{*}\right)\right)^{\prime}}+\right. \\
\left.+\cdots+\frac{g(z)-g\left(z_{*}\right)}{n g^{\prime}(z) /\left({ }^{[n-1]} f_{g}\left(z_{*}\right)\right)^{\prime}}+\frac{g(z)-g\left(z_{*}\right)}{R_{n}(g ; z)}\right)^{-1}
\end{gathered}
$$

where $R_{n}(g ; z)$ is remainder term.

\section{Rules of reciprocal $g$-differentiation $2-$ nd type}

We define the rules of the reciprocal $g$-differentiation of 2-nd type to the sum, difference, product and quotient two functions. Let all functions $f(z), h(z), f_{1}(z), f_{2}(z), \ldots, f_{n}(z)$ have in the point of compact $\mathcal{Z} \subset \mathbb{C}$ finite nonzero reciprocal $g$-derivatives of 2 -nd type.

Theorem 5. Let reciprocal g-derivatives of 2-nd type functions $u=f(z)$ and $v=h(z)$ exists. Reciprocal $g$-derivatives of 2-nd type to the sum, difference, prouct and quotient of this functions are determined by formulas

$$
\begin{aligned}
{ }^{[1]}(u \pm v)_{g} & =\frac{(u \pm v)^{2} \cdot{ }^{[1]} v_{g} \cdot{ }^{[1]} u_{g}}{u^{2} \cdot{ }^{[1]} v_{g} \pm v^{2} \cdot{ }^{[1]} u_{g}} \\
{ }^{[1]}(u v)_{g} & =\frac{u v \cdot{ }^{[1]} u_{g} \cdot{ }^{[11} v_{g}}{u \cdot{ }^{[1]} v_{g}+v \cdot{ }^{[1]} u_{g}} \\
{ }^{[1]}(u / v)_{g} & =\frac{(u / v) \cdot{ }^{[1]} u_{g} \cdot{ }^{[1]} v_{g}}{u \cdot{ }^{[1]} v_{g}-v \cdot{ }^{[1]} u_{g}}
\end{aligned}
$$

Proof. From (14) follows

$$
{ }^{[1]}(u \pm v)_{g}=-\frac{(u \pm v)^{2} g^{\prime}}{u^{\prime} \pm v^{\prime}}=\frac{-(u \pm v)^{2} g^{\prime}}{\frac{-u^{2} g^{\prime}}{{ }^{[1]} u_{g}} \pm \frac{-v^{2} g^{\prime}}{{ }^{[1]} v_{g}}}=\frac{(u \pm v)^{2} \cdot{ }^{[1]} v_{g} \cdot{ }^{[1]} u_{g}}{u^{2} \cdot{ }^{[1]} v_{g} \pm v^{2} \cdot{ }^{[1]} u_{g}}
$$

Similarly

$$
\begin{gathered}
{ }^{[1]}(u \cdot v)_{g}=\frac{-(u \cdot v)^{2} g^{\prime}}{u^{\prime} \cdot v+u \cdot v^{\prime}}=\frac{-(u v)^{2} g^{\prime}}{\frac{-v u^{2} g^{\prime}}{{ }^{[1]} u_{g}}+\frac{-u v^{2} g^{\prime}}{{ }^{[1]} v_{g}}}=\frac{u v \cdot{ }^{[1]} u_{g} \cdot{ }^{[1]} v_{g}}{u \cdot{ }^{[1]} v_{g}+v \cdot{ }^{[1]} u_{g}}, \\
{ }^{[1]}(u / v)_{g}=\frac{-(u / v)^{2} g^{\prime}}{\frac{u^{\prime} v-u v^{\prime}}{v^{2}}}=\frac{-u^{2} \cdot g^{\prime}}{\frac{-v u^{2} g^{\prime}}{u v^{2} g^{\prime}}}=\frac{(u / v) \cdot{ }^{[1]} u_{g} \cdot{ }^{[1]} v_{g}}{u \cdot{ }^{[1]} v_{g}-v \cdot{ }^{[1]} u_{g}}
\end{gathered}
$$

Formulas (18)-(20) have been proved. 
Theorem 6. If the functions $f_{k}(z), k=1,2, \ldots, n$, have reciprocal $g$-derivatives of 2 -nd type then

$$
\begin{aligned}
{ }^{[1]}\left(\sum_{k=1}^{n} f_{k}(z)\right)_{g} & =\frac{\left(\sum_{k=1}^{n} f_{k}(z)\right)^{2} \prod_{k=1}^{n}{ }^{[1]}\left(f_{k}(z)\right)_{g}}{\sum_{k=1}^{n} f_{k}^{2}(z) \prod_{\substack{j=1 \\
j \neq k}}^{n}\left(f_{j}(z)\right)_{g}}, \\
{ }^{[1]}\left(\prod_{k=1}^{n} f_{k}(z)\right)_{g} & =\frac{\prod_{k=1}^{n} f_{k}(z) \cdot{ }^{[1]}\left(f_{k}(z)\right)_{g}}{\sum_{k=1}^{n} f_{k}(z) \cdot \prod_{\substack{j=1 \\
j \neq k}}^{n}\left[f_{j}(z)\right)_{g}} .
\end{aligned}
$$

Proof. Formulas (21)-(22) are proved by induction. Formula (21) is true when $n=2$. For $n=3$, from (18) we have

$$
\begin{gathered}
{ }^{[1]}\left(f_{1}(z)+f_{2}(z)+f_{3}(z)\right)_{g}=\frac{\left(f_{1}(z)+f_{2}(z)+f_{3}(z)\right)^{2} \cdot{ }^{[1]}\left(f_{1}(z)+f_{2}(z)\right)_{g} \cdot{ }^{[1]}\left(f_{3}(z)\right)_{g}}{\left(f_{1}(z)+f_{2}(z)\right)^{2} \cdot{ }^{[1]}\left(f_{3}(z)\right)_{g}+f_{3}^{2}(z) \cdot{ }^{[1]}\left(f_{1}(z)+f_{2}(z)\right)_{g}}= \\
=\frac{\left(f_{1}(z)+f_{2}(z)+f_{3}(z)\right)^{2} \cdot \frac{\left(f_{1}(z)+f_{2}(z)\right)^{2} \cdot{ }^{[1]}\left(f_{1}(z)\right)_{g} \cdot{ }^{[1]}\left(f_{2}(z)\right)_{g}}{\left.f_{1}^{2}(z) \cdot{ }^{[1]}\left(f_{2}(z)\right)_{g}+f_{2}^{2}(z) \cdot f_{3}(z)\right)_{g}\left(f_{1}(z)\right)_{g}}}{\left(f_{1}(z)+f_{2}(z)\right)^{2} \cdot{ }^{[1]}\left(f_{3}(z)\right)_{g}+f_{3}^{2}(z) \cdot \frac{\left(f_{1}(z)+f_{2}(z)\right)^{2} \cdot{ }^{[1]}\left(f_{1}(z)\right)_{g} \cdot{ }^{[1]}\left(f_{2}(z)\right)_{g}}{f_{1}^{2}(z) \cdot{ }^{[1]}\left(f_{2}(z)\right)_{g}+f_{2}^{2}(z) \cdot{ }^{[1]}\left(f_{1}(z)\right)_{g}}}= \\
=\frac{\left(f_{1}(z)+f_{2}(z)+f_{3}(z)\right)^{2} \cdot{ }^{[1]}\left(f_{1}(z)\right)_{g} \cdot{ }^{[1]}\left(f_{2}(z)\right)_{g} \cdot{ }^{[1]}\left(f_{3}(z)\right)_{g}}{f_{1}^{2}(z) \cdot{ }^{[1]}\left(f_{2}(z)\right)_{g} \cdot{ }^{[1]}\left(f_{3}(z)\right)_{g}+f_{2}^{2} \cdot{ }^{[1]}\left(f_{1}(z)\right)_{g} \cdot{ }^{[1]}\left(f_{3}(z)\right)_{g}+f_{3}^{2}(z) \cdot{ }^{[1]}\left(f_{1}(z)\right)_{g} \cdot{ }^{[1]}\left(f_{2}(z)\right)_{g}} .
\end{gathered}
$$

Suppose that (21) is executed when $n=m-1$. Then

$$
\begin{aligned}
& { }^{[1]}\left(\sum_{k=1}^{m} f_{k}(z)\right)_{g}=\frac{\left(\sum_{k=1}^{m} f_{k}(z)\right)^{2} \cdot{ }^{[1]}\left(\sum_{k=1}^{m-1} f_{k}(z)\right)_{g} \cdot{ }^{[1]}\left(f_{m}(z)\right)_{g}}{\left(\sum_{k=1}^{m-1} f_{k}(z)\right)^{2} \cdot{ }^{[1]}\left(f_{m}(z)\right)_{g}+f_{m}^{2}(z) \cdot{ }^{[1]}\left(\sum_{k=1}^{m-1} f_{k}(z)\right)_{g}}=
\end{aligned}
$$

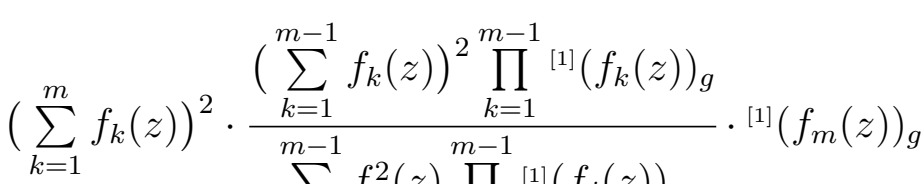

$$
\begin{aligned}
& =\frac{\sum_{k=1} f_{k}^{2}(z) \prod_{\substack{j=1 \\
j \neq k}}^{[1]}\left(f_{j}(z)\right)_{g}}{\left(\sum_{k=1}^{m-1} f_{k}(z)\right)^{2} \cdot{ }^{[1]}\left(f_{m}(z)\right)_{g}+f_{m}^{2}(z) \cdot \frac{\left(\sum_{k=1}^{m-1} f_{k}(z)\right)^{2} \prod_{k=1}^{m-1}{ }^{[1]}\left(f_{k}(z)\right)_{g}}{\sum_{k=1}^{m-1} f_{k}^{2}(z) \prod^{m-1}{ }^{[1]}\left(f_{j}(z)\right)_{g}}}=\frac{\left(\sum_{k=1}^{m} f_{k}(z)\right)^{2} \prod_{k=1}^{m} f_{k}^{2}(z) \prod_{\substack{j=1 \\
j \neq k}}^{m}\left(f_{k}(z)\right)_{g}\left(f_{j}(z)\right)_{g}}{\sum_{k=1}^{m}} . \\
& \sum_{k=1}^{m-1} f_{k}^{2}(z) \prod_{\substack{j=1 \\
j \neq k}}^{m-1}\left(f_{j}(z)\right)_{g}
\end{aligned}
$$


Similarly, if $n=2$ then formula (22) is true. From (19) follows when $n=3$

$$
\begin{gathered}
{ }^{[1]}\left(f_{1}(z) f_{2}(z) f_{3}(z)\right)_{g}=\frac{f_{1}(z) f_{2}(z) f_{3}(z) \cdot{ }^{[1]}\left(f_{1}(z) f_{2}(z)\right)_{g} \cdot{ }^{[1]}\left(f_{3}\right)_{g}}{f_{1}(z) f_{2}(z) \cdot{ }^{[1]}\left(f_{3}(z)\right)_{g}+f_{3}(z) \cdot{ }^{[1]}\left(f_{1}(z) f_{2}(z)\right)_{g}}= \\
=\frac{f_{1}(z) f_{2}(z) f_{3}(z) \cdot \frac{f_{1}(z) f_{2}(z) \cdot{ }^{[1]}\left(f_{1}(z)\right)_{g} \cdot{ }^{[1]}\left(f_{2}(z)\right)_{g}}{f_{1}(z) \cdot{ }^{[1]}\left(f_{2}(z)\right)_{g}+f_{2} \cdot{ }^{[1]}\left(f_{1}(z)\right)_{g}}\left(f_{3}\right)_{g}}{f_{1}(z) f_{2}(z) \cdot{ }^{[1]}\left(f_{3}(z)\right)_{g}+f_{3}(z) \cdot \frac{f_{1}(z) f_{2}(z) \cdot{ }^{[1]}\left(f_{1}(z)\right)_{g} \cdot{ }^{[1]}\left(f_{2}(z)\right)_{g}}{f_{1}(z) \cdot{ }^{[1]}\left(f_{2}(z)\right)_{g}+f_{2} \cdot{ }^{[1]}\left(f_{1}(z)\right)_{g}}}= \\
=\frac{f_{1}(z) f_{2}(z) f_{3}(z) \cdot{ }^{[1]}\left(f_{1}(z)\right)_{g} \cdot{ }^{[1]}\left(f_{2}(z)\right)_{g} \cdot{ }^{[1]}\left(f_{3}\right)_{g}}{f_{1}(z) \cdot{ }^{[1]}\left(f_{2}(z)\right)_{g} \cdot{ }^{[1]}\left(f_{3}(z)\right)_{g}+f_{2}(z) \cdot{ }^{[1]}\left(f_{1}(z)\right)_{g} \cdot{ }^{11]}\left(f_{3}(z)\right)_{g}+f_{3}(z) \cdot{ }^{[1]}\left(f_{1}(z)\right)_{g} \cdot{ }^{[1]}\left(f_{2}(z)\right)_{g}} .
\end{gathered}
$$

Suppose that (22) holds for $n=m-1$. Then from (19) we have

$$
\begin{gathered}
{ }^{[1]}\left(\prod_{k=1}^{m} f_{k}(z)\right)_{g}=\frac{\prod_{k=1}^{m} f_{k}(z) \cdot{ }^{[1]}\left(\prod_{k=1}^{m-1} f_{k}(z)\right)_{g} \cdot{ }^{[1]}\left(f_{m}(z)\right)_{g}}{\prod_{k=1}^{m-1} f_{k}(z) \cdot{ }^{[1]} f_{m}(z)+f_{m}(z) \cdot{ }^{[1]}\left(\prod_{k=1}^{m-1} f_{k}(z)\right)_{g}} \\
=\frac{\prod_{k=1}^{m} f_{k}(z) \cdot \frac{\prod_{k=1}^{m-1} f_{k}(z) \cdot{ }^{[1]}\left(f_{k}(z)\right)_{g}}{\sum_{k=1}^{m-1} f_{k}(z) \cdot \prod_{\substack{j=1 \\
j \neq k}}^{m-1}{ }^{[1]}\left(f_{j}(z)\right)_{g}}{ }^{[1]}\left(f_{m}(z)\right)_{g}}{\prod_{k=1}^{m-1} f_{k}(z) \cdot{ }^{[1]} f_{m}(z)+f_{m}(z) \cdot \frac{\prod_{k=1}^{m-1} f_{k}(z) \cdot{ }^{[1]}\left(f_{k}(z)\right)_{g}}{\sum_{k=1}^{m-1} f_{k}(z) \cdot \prod_{\substack{j=1 \\
j \neq k}}^{m-1}{ }^{[1]}\left(f_{j}(z)\right)_{g}}}=\frac{\prod_{k=1}^{m} f_{k}(z) \cdot{ }^{[1]}\left(f_{k}(z)\right)_{g}}{\sum_{k=1}^{m} f_{k}(z) \cdot \prod_{\substack{j=1 \\
j \neq k}}^{m}{ }^{[1]}\left(f_{j}(z)\right)_{g}} .
\end{gathered}
$$

Theorem 7. Let function $f(z)$ has reciprocal g-derivative of 2 -nd type of $n$-th order, $n=0,1, \ldots$, for arbitrary $z \in \mathcal{Z}$ and $C$ is constant then

$$
{ }^{[2 n]}(C f(z))_{g}=\frac{1}{C} \cdot{ }^{[2 n]} f_{g}(z),{ }^{[2 n+1]}(C f(z))_{g}=C \cdot{ }^{[2 n+1]} f_{g}(z) .
$$

Proof. We prove the theorem by induction. It is easy to see that

$$
{ }^{[0]}(C f(z))_{g}=\frac{1}{C} \cdot{ }^{[0]} f_{g}(z),{ }^{[1]}(C f(z))_{g}=C \cdot{ }^{[1]} f_{g}(z),{ }^{[2]}(C f(z))_{g}=\frac{1}{C} \cdot{ }^{[2]} f_{g}(z) .
$$

Suppose that the statement of the theorem is valid to $n=k$. Then, from recurrence formula (17) we have that for $n=k+1$

$$
\begin{gathered}
{ }^{[2 k+2]}(C f(z))_{g}=\frac{(2 k+2) g^{\prime}(z)}{\left({ }^{[2 k+1]}(C f(z))_{g}\right)^{\prime}}+{ }^{[2 k]}(C f(z))=\frac{1}{C} \cdot{ }^{[2 k+2]} f_{g}(z) . \\
{ }^{[2 k+3]}(C f(z))_{g}=\frac{(2 k+3) g^{\prime}(z)}{\left({ }^{[2 k+2]}(C f(z))_{g}\right)^{\prime}}+{ }^{[2 k+1]}(C f(z))_{g}=C \cdot{ }^{[2 k+3]} f_{g}(z) .
\end{gathered}
$$

Theorem is valid at arbitrary $n$. 


\section{References}

[1] Thiele T.N., Interpolationsprechnung, Commisission von B.G. Teubner, 1909.

[2] M.M. Pahirya, Expansion of functions of complex variable in the Thiele-like quasi-inverse continued fraction, Scien. Bull. of Uzhhorod Univ. Series of Math. and Informath. 25 (2014) 131-144. (in Ukrainian)

[3] M.M. Pahirya, Approximation functions by continued fractions, Grazhda, Uzhhorod, 2016. (in Ukrainian)

[4] W.B. Jones, W.J. Thron, Continued fractions, analytic theory and applications, Encyclopedia of Mathematics and its Applications, Wesley, 1980. 